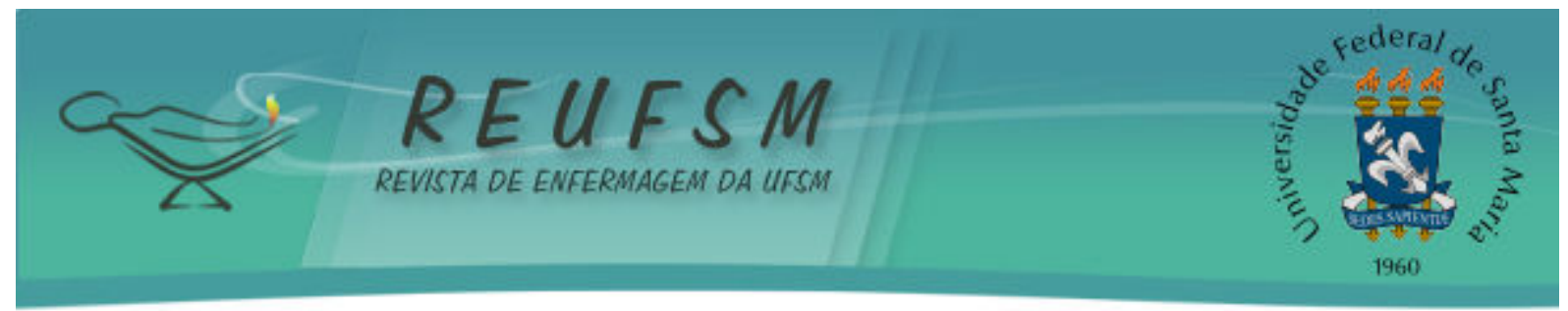

ARTIGO ORIGINAL

\title{
PERFIL DOS CASOS DE SÍNDROME DA IMUNODEFICIÊNCIA ADQUIRIDA EM UM ESTADO DO NORDESTE DO BRASIL*
}

\section{PROFILE OF THE CASES OF ACQUIRED IMMUNE DEFICIENCY SYNDROME IN A STATE OF NORTHEASTERN BRAZIL}

\section{PERFIL DE LOS CASOS DE SÍNDROME DE INMUNODEFICIENCIA ADQUIRIDA EN UN ESTADO DEL NORDESTE DE BRASIL}

\author{
Ilisdayne Thallita Soares da Silva ${ }^{1}$ \\ Dalva Cezar da Silva² \\ Marina de Góes Salvetti $i^{3}$ \\ Gilson de Vasconcelos Torres ${ }^{4}$ \\ Richardson Augusto Rosendo da Silva ${ }^{5}$ \\ Nilba Lima de Souza ${ }^{6}$
}

\section{Doi: $10.5902 / 2179769215207$}

RESUMO: Objetivo: descrever o perfil dos casos de Síndrome da Imunodeficiência Adquirida no Rio Grande do Norte, Brasil, no período de 1980 a 2013. Método: estudo quantitativo e descritivo com dados coletados em maio de 2014, por meio do Departamento de Informática do Sistema Único de Saúde. Resultados: foram diagnosticados 4.666 casos no período analisado, sendo $66,6 \%$ do sexo masculino, $82,6 \%$ da faixa etária entre 20 e 49 anos, $31,6 \%$ da raça parda e com predomínio de escolaridade baixa. A categoria de exposição mais frequente foi a de heterossexuais, tanto para o sexo masculino quanto para o sexo feminino. Conclusão: no Rio Grande do Norte, no período estudado, o perfil epidemiológico apresentou predomínio de pessoas do sexo masculino, em idade economicamente ativa e com baixa escolaridade. Conhecer essas características epidemiológicas pode contribuir para o planejamento de ações estratégicas de saúde envolvendo os profissionais de saúde e a enfermagem.

Descritores: Síndrome da imunodeficiência adquirida; Epidemiologia; Perfil de saúde; Sistemas de informação; Enfermagem.

ABSTRACT: Objective: to describe the profile of the cases of Acquired Immunodeficiency Syndrome in Rio Grande do Norte, Brazil, from 1980 to 2013. Method: descriptive and quantitative study, in which data was collected in May 2014 by the Computer Department of the Unified Health System. Results: 4666 cases were diagnosed in the period analyzed,

\footnotetext{
* Artigo realizado na Disciplina de Vigilância à Saúde, do Programa de Pós-Graduação em Enfermagem, da Universidade Federal do Rio Grande do Norte (UFRN).

${ }^{1}$ Enfermeira da Estratégia Saúde da Família, Prefeitura Municipal de Cuité. Mestranda em Enfermagem do Programa de Pós-Graduação em Enfermagem da UFRN. Cuité, Paraíba, Brasil. E-mail: ilisdayne@yahoo.com.br

2 Enfermeira do Departamento de Enfermagem da Universidade Federal de Santa Maria (UFSM). Doutoranda em Enfermagem do Programa de Pós-Graduação em Enfermagem da UFRN. Santa Maria, Rio Grande do Sul, Brasil. E-mail: dalvacezarsilva@yahoo.com.br

3 Enfermeira. Pós-Doutora em Enfermagem - UFRN. Professora Doutora da Escola de Enfermagem da Universidade de São Paulo (USP). São Paulo, SP, Brasil. E-mail: mgsalvetti@usp.br

${ }^{4}$ Enfermeiro. Pós-Doutor em Enfermagem - Évora/Portugal. Doutor em Enfermagem. Professor Titular do Departamento de Enfermagem da UFRN. Bolsista de Produtividade em Pesquisa PQ2/CNPq. Natal, RN, Brasil. Email: gilsonvtorres@hotmail.com

${ }^{5}$ Enfermeiro. Doutor em Ciências da Saúde. Docente do Departamento de Enfermagem da UFRN. Natal, RN, Brasil. Natal, RN, Brasil. E-mail: rirosendo@yahoo.com.br

${ }^{6}$ Enfermeira. Doutora em Ciências da Saúde. Docente do Departamento de enfermagem da UFRN. Natal, RN, Brasil. E-mail: nilbalima@ufrnet.br
} 


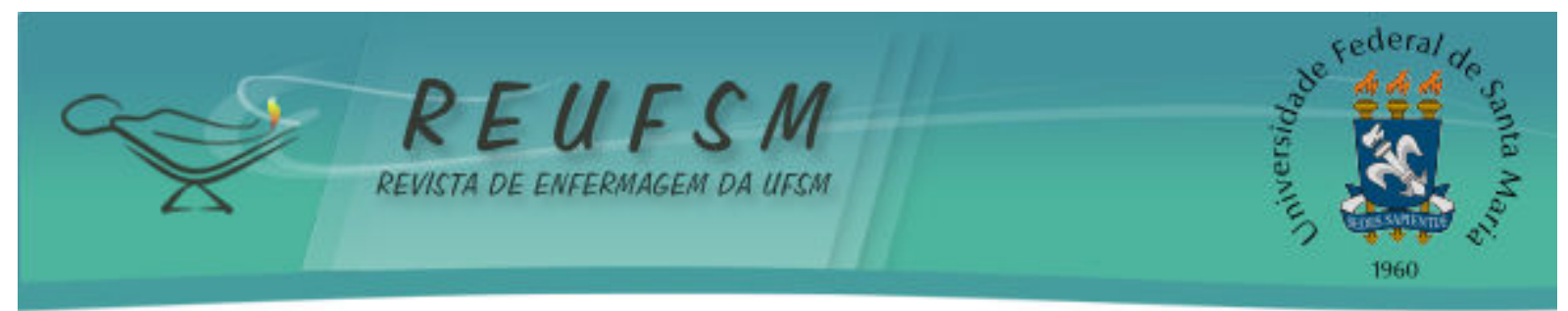

from which $66.6 \%$ were male, $82.6 \%$ between 20 and 49 years old, $31.6 \%$ were pardos and predominantly with low education level. The category with most common exposure was the heterosexuals, both for males and females. Conclusion: in Rio Grande do Norte, during the period studied, the epidemiological profile showed a predominance of males in economically active age and with low level of education. Identifying these epidemiological characteristics can contribute to the strategic planning of health actions involving health and nursing professionals.

Descriptors: Acquired immunodeficiency syndrome; Epidemiology; Health profile; Information systems; Nursing.

RESUMEN: Objetivo: describir el perfil de los casos de Síndrome de Inmunodeficiencia Adquirida en Rio Grande do Norte (Brasil), 1980-2013. Método: estudio cuantitativo y descriptivo, con datos recogidos en mayo de 2014 por el Departamento de Informática del Sistema Único de Salud. Resultados: 4.666 casos fueron diagnosticados en el período de estudio, con el $66,6 \%$ hombres, $82,6 \%$ del grupo de edad entre 20 y 49 años, 31,6\% mulato y predominio de bajo nivel de educación. La categoría de exposición más frecuente fue la heterosexual, tanto para varones como para mujeres. Conclusión: en Rio Grande do Norte el perfil epidemiológico mostró un predominio de varones en edad económicamente activa y baja escolarización. Conocer estas características epidemiológicas pueden contribui a la planificación estratégica de las acciones de salud que involucran profesionales de salud y de enfermería.

Descriptores: Síndrome de inmunodeficiencia adquirida; Epidemiología; Perfil de salud; Sistemas de información; Enfermería.

\section{INTRODUÇÃO}

A epidemia da Síndrome da Imunodeficiência Adquirida (AIDS) continua sendo um grave problema de saúde pública, fazendo milhares de vítimas todos os anos e impondo desafios à comunidade científica mundial. Estudo que analisou a prevalência, a incidência e as tendências da infecção pelo Vírus da Imunodeficiência Humana (HIV) na América Latina e Caribe apontou que a falta de informação, a prevenção e o cuidado para o HIV ainda são importantes questões a serem resolvidas. ${ }^{1}$

Os dados numéricos retratam esta evolução, pois já foram notificados 686.478 casos de AIDS no Brasil desde a identificação do primeiro caso, em meados da década de 1980, até junho de 2013, dos quais 445.197 (64,9\%) são do sexo masculino e 241.223 $(35,1 \%)$ do sexo feminino. ${ }^{2}$

Quando surgiram os primeiros casos de AIDS, estes se restringiam a um grupo considerado de maior risco para a infecção: profissionais do sexo, homossexuais e usuários de drogas injetáveis (UDI). Entretanto, o aumento do número de pessoas acometidas dentro dos que não pertenciam ao grupo de risco, evidenciou a mudança no perfil epidemiológico, com a identificação da doença em mulheres heterossexuais e com relacionamento estável. ${ }^{3}$

Identifica-se, no Brasil e no mundo, a diminuição da morbidade e da mortalidade pela AIDS, decorrente principalmente dos tratamentos eficazes, porém ainda existe a antiga visão de discriminação e preconceito. Esse quadro, muitas vezes, no processo de cuidar dos profissionais da saúde pode contribuir para o aumento da vulnerabilidade de gênero no contexto da feminização dos casos de AIDS. ${ }^{4}$

No cenário atual, o Brasil apresenta a epidemia da AIDS estável e concentrada em alguns subgrupos populacionais em situação de vulnerabilidade. ${ }^{2}$ Essa doença já não está mais restrita a "grupos de risco" específicos, tendo uma velocidade de crescimento do 


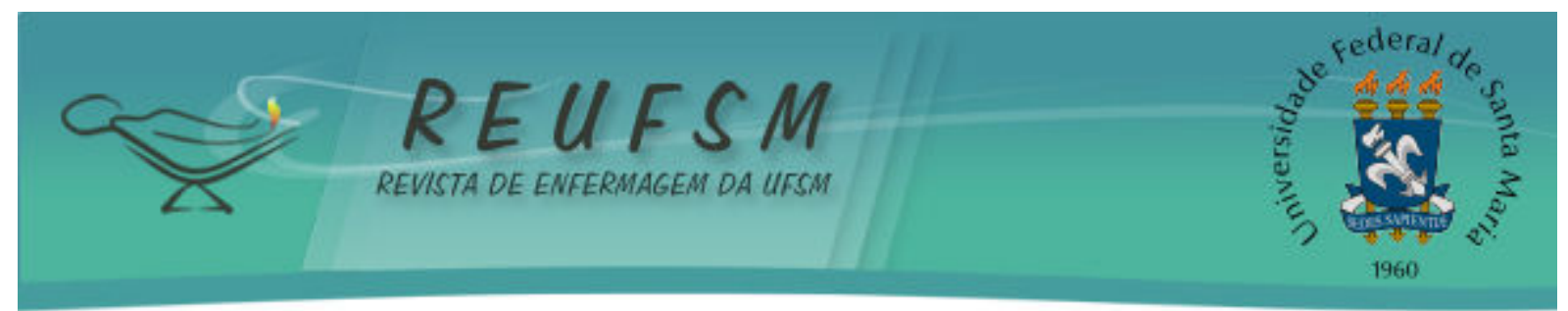

número de novos casos mais expressiva entre mulheres e entre aqueles com piores condições sociais. $^{5}$

Ao analisar o perfil da epidemia de AIDS no contexto brasileiro relacionando-o à organização do setor saúde, às situações relacionadas ao risco de infecção e ao grau e implantação da resposta, constatou-se diferenças entre as regiões. As regiões Sudeste, Sul e os maiores centros urbanos do país, são caracterizados por apresentar maior controle da doença. Por outro lado, as regiões Norte e Nordeste e os municípios de médio porte apresentam a epidemia da AIDS em crescimento e capacidade de resposta relativamente baixa. ${ }^{6}$

Com relação à magnitude e à tendência da doença, ressalta-se que os municípios com epidemias de AIDS de pequena magnitude que apresentam crescimento acima da média nacional, localizam-se no interior dos estados, com destaque para o Estado do Rio Grande do Norte.

Sob essa perspectiva formulou-se a seguinte questão norteadora: Como se apresenta o perfil epidemiológico dos casos de AIDS no Rio Grande do Norte, Brasil, no período de 1980 a 2013 ?

Esse estudo justifica-se pela necessidade de conhecer o perfil epidemiológico dos casos de AIDS no Estado do Rio Grande do Norte, a fim de proporcionar uma reflexão crítica sobre o problema nessa região. Além disso, permite uma compreensão mais profunda da realidade local para que se possa formular estratégias e implementar programas de prevenção, promoção e controle do HIV/AIDS. Tais informações poderão ser utilizadas para direcionar políticas públicas de saúde, além de instrumentalizar a enfermagem e outros profissionais de saúde para abordar essa população, trazendo melhorias na qualidade da assistência prestada a esses pacientes.

Assim, objetivou-se descrever o perfil dos casos de AIDS no Rio Grande do Norte, Brasil, no período de 1980 a 2013.

\section{MÉTODO}

Trata-se de um estudo quantitativo, descritivo com os dados referentes à série histórica de 1980 a 2013. A coleta dos dados ocorreu em maio de 2014 , sendo realizada a partir da fonte secundária de dados de domínio público do Ministério da Saúde Departamento de Informática do Sistema Único de Saúde (DATASUS), disponível no endereço eletrônico: www.datasus.gov.br. Os dados encontravam-se consolidados até 30/06/2013 e incluíram-se somente os casos notificados de AIDS.

Nesta pesquisa, foi utilizada uma amostra com AIDS do Estado do Rio Grande do Norte, da zona urbana e rural, com menos de um ano de idade até a idade acima de oitenta anos. Os dados coletados são provenientes do Sistema de Informação de Agravos de Notificação (SINAN), Sistema de Informação sobre Mortalidade (SIM) e Sistema de Controle de Exames Laboratoriais da Rede Nacional de Contagem de Linfócitos CD4+/CD8+ e Carga Viral (SISCEL).

Localizado na região Nordeste do Brasil, o Estado do Rio Grande do Norte é constituído por 167 municípios, a área total é de $52.811,047 \mathrm{~km}^{2}$ e em 2013 apresentou uma população estimada de 3.373 .960 habitantes $^{7}$.

Nesta pesquisa, as variáveis avaliadas foram selecionadas a partir das categorias estabelecidas pelo DATASUS, sendo as seguintes: frequência da AIDS; ano diagnóstico; sexo (Masculino, Feminino), faixa etária, escolaridade (Analfabeto, Ensino Fundamental, Ensino Médio, Ensino Superior, Não se aplica, Ignorado), raça/cor (Branca, Preta, Amarela, Parda, Indígena, Ignorada) e categoria de exposição (Heterossexual, Homossexual, Bissexual, UDI, Hemofílico, Transfusão, Transmissão vertical, Ignorado). 


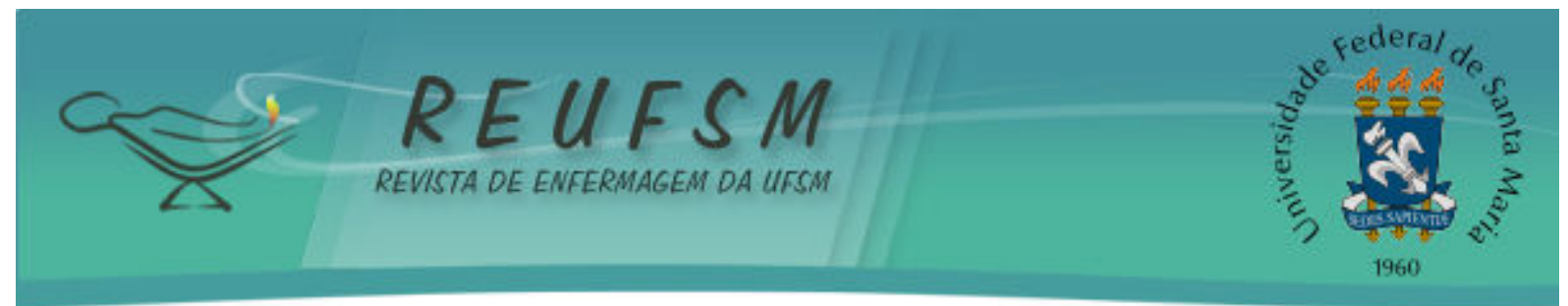

A tabulação dos dados foi realizada por meio do software TabNetwin 32 2.4. As tabelas foram exportadas para o programa Microsoft Excel ${ }^{\circledR}$ 2010, no qual foram construídos gráficos e duas tabelas para a apresentação dos resultados. Os dados foram analisados por meio de estatística descritiva simples e apresentados em valores absolutos e percentuais.

Por se tratar de um banco de domínio público e de livre acesso, não foi necessário submeter o projeto ao Comitê de Ética em Pesquisa.

\section{RESULTADOS}

A análise dos dados mostrou que no período de 1980 a 2013 foram notificados 4.666 casos de AIDS no Estado do Rio Grande do Norte. A incidência da doença apresentouse crescente entre 1980 e 2012, com alguns períodos de leve queda e redução importante entre 2012 e 2013, passando de 407 casos para 192 casos (Figura 1).

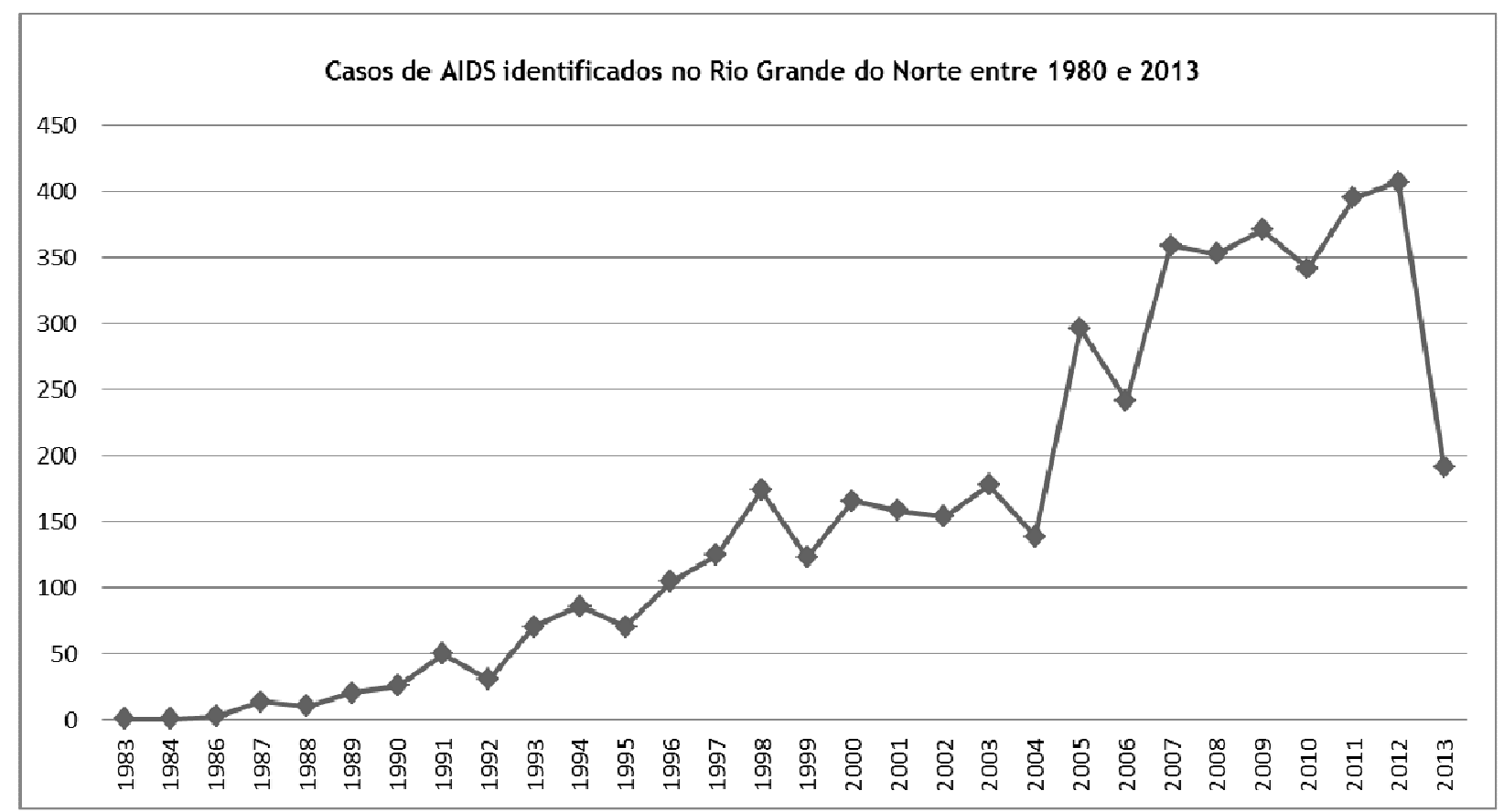

Figura 1- Evolução do número de casos de AIDS no Rio Grande do Norte entre 1980 e 2013.

Em relação ao sexo, $66,6 \%$ dos casos eram do sexo masculino e 33,4\% feminino. Quanto à evolução dos casos de AIDS, segundo sexo no Rio Grande do Norte, nota-se que o sexo masculino foi mais afetado pela doença em todo o período analisado com diferença mais expressiva entre os sexos nos últimos 10 anos (Figura 2). 

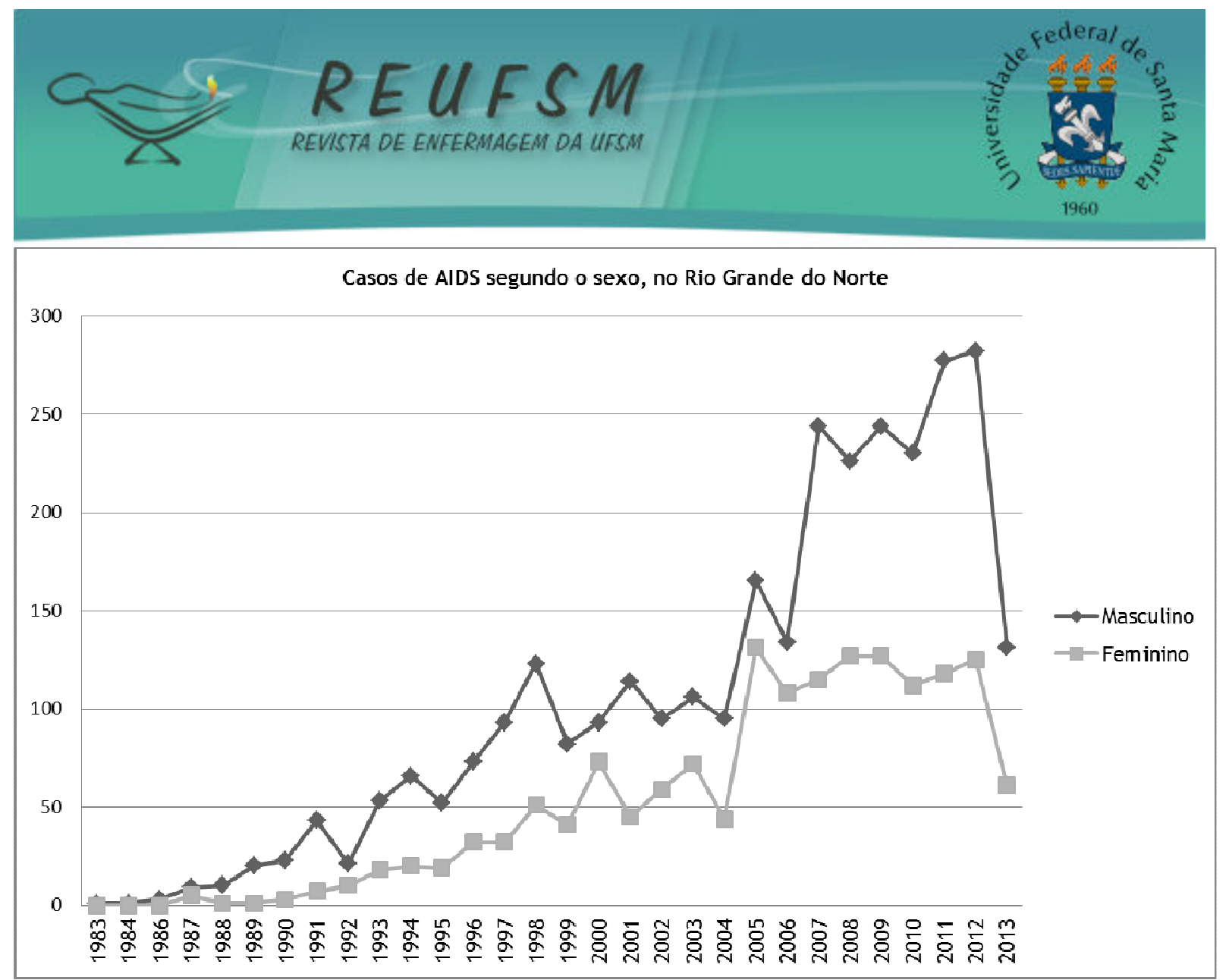

Figura 2- Evolução do número de casos de AIDS segundo sexo no Rio Grande do Norte, entre 1980 e 2013. 


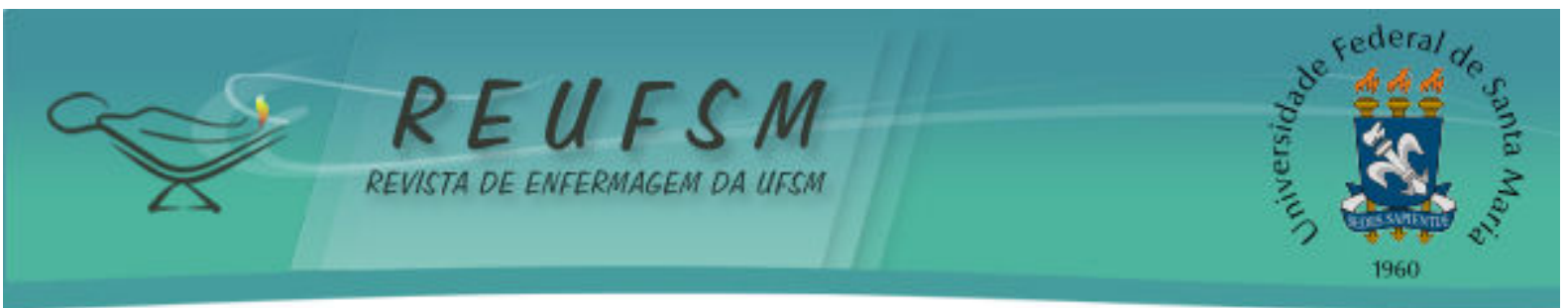

$\mathrm{Na}$ Tabela 1, apresentam-se os casos de AIDS, segundo variáveis sociodemográficas no Rio Grande do Norte, no período de 1980 a 2013.

Tabela 1 - Casos de AIDS, segundo variáveis sociodemográficas no Rio Grande do Norte, no período de 1980 a 2013.

\begin{tabular}{|c|c|c|c|c|c|c|}
\hline \multirow{2}{*}{ Variáveis } & \multicolumn{2}{|c|}{ Masculino $(n=3109)$} & \multicolumn{2}{|c|}{ Feminino $(n=1557)$} & \multicolumn{2}{|c|}{ Total $(n=4.666)$} \\
\hline & $n$ & $\%$ & $\mathrm{n}$ & $\%$ & $\mathrm{n}$ & $\%$ \\
\hline \multicolumn{7}{|l|}{ Faixa etária (anos) } \\
\hline Menor de 1 a 19 & 100 & 3,2 & 120 & 7,7 & 220 & 4,7 \\
\hline 20 a 49 & 2600 & 83,6 & 1256 & 80,7 & 3856 & 82,6 \\
\hline 50 a 79 & 402 & 12,9 & 179 & 11,5 & 581 & 12,4 \\
\hline 80 e mais & 5 & 0,2 & 2 & 0,1 & 7 & 0,2 \\
\hline Ignorado & 2 & 0,1 & 0 & 0 & 2 & 0,1 \\
\hline \multicolumn{7}{|l|}{ Escolaridade } \\
\hline Analfabeto & 129 & 4,2 & 76 & 4,9 & 205 & 4,4 \\
\hline Ensino Fundamental* & 771 & 24,8 & 423 & 27,2 & 1194 & 25,7 \\
\hline Ensino Médio* & 445 & 14,3 & 162 & 10,4 & 607 & 13,0 \\
\hline Ensino Superior* & 232 & 7,5 & 35 & 2,2 & 267 & 5,8 \\
\hline Não se aplica & 18 & 0,5 & 27 & 1,7 & 45 & 0,1 \\
\hline Ignorado & 1514 & 48,7 & 834 & 53,6 & 2348 & 51,0 \\
\hline \multicolumn{7}{|l|}{ Raça/cor } \\
\hline Branca & 454 & 14,6 & 246 & 15,6 & 700 & 15,0 \\
\hline Negra & 87 & 2,8 & 59 & 3,7 & 146 & 3,2 \\
\hline Amarela & 5 & 0,2 & 8 & 0,5 & 13 & 0,2 \\
\hline Parda & 1019 & 32,7 & 456 & 29,2 & 1475 & 31,6 \\
\hline Indígena & 3 & 0,1 & 1 & 0,6 & 4 & 0,1 \\
\hline Ignorado & 1541 & 49,6 & 787 & 50,4 & 2328 & 49,9 \\
\hline
\end{tabular}

*completo ou incompleto.

$\mathrm{Na}$ Tabela 1 pode-se verificar que a faixa etária mais afetada pela AIDS ficou entre 20 e 49 anos. A incidência foi menor nas idades mais avançadas e foi muito baixa entre os mais jovens e as crianças.

Em relação à escolaridade, identificou-se predomínio de indivíduos com ensino fundamental completo ou incompleto $(25,7 \%)$, seguido de ensino médio completo ou incompleto $(13,0 \%)$. Quanto à raça notou-se predomínio de indivíduos pardos $(31,6 \%)$ seguidos de brancos $(15,0 \%)$ e negros $(3,2 \%)$ (Tabela 1$)$.

$\mathrm{Na}$ Tabela 2, apresentam-se os casos de AIDS, segundo categoria de exposição no Rio Grande do Norte, no período de 1980 a 2013. 


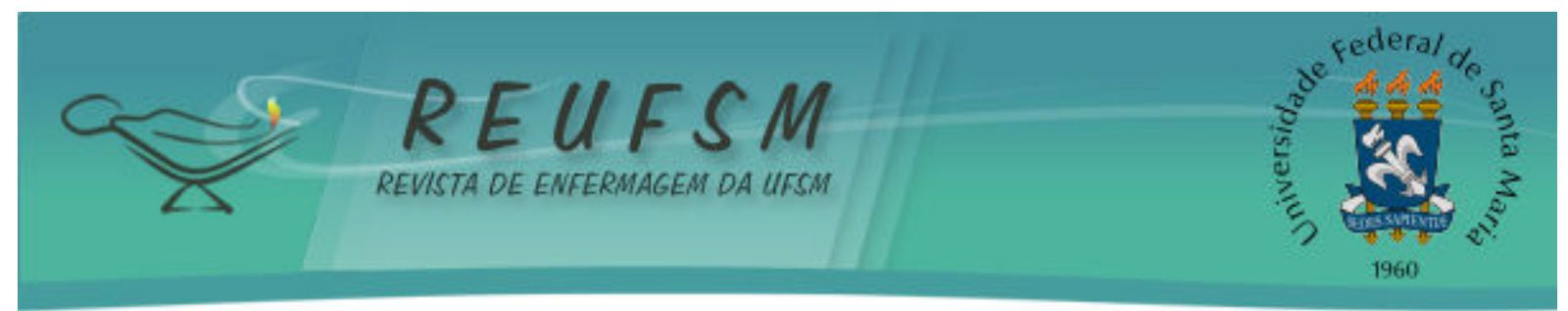

Tabela 2- Casos de AIDS, segundo categoria de exposição no Rio Grande do Norte, no período de 1980 a 2013.

\begin{tabular}{ccccccc}
\hline Categoria de Exposição & \multicolumn{2}{c}{ Masculino $\mathbf{( n = 3 1 0 9 )}$} & \multicolumn{2}{c}{ Feminino $\mathbf{( n = 1 5 5 7 )}$} & \multicolumn{2}{c}{ Total $(\mathbf{n = 4 . 6 6 6 )}$} \\
& $\mathbf{n}$ & $\mathbf{\%}$ & $\mathbf{n}$ & $\%$ & $\mathbf{n}$ & $\mathbf{\%}$ \\
\hline Homossexual & 708 & 22,8 & 0 & 0 & 708 & 15,1 \\
Bissexual & 394 & 12,7 & 0 & 0 & 394 & 8,3 \\
Heterossexual & 831 & 26,8 & 995 & 63,9 & 1826 & 39,1 \\
UDI* & 86 & 2,7 & 27 & 1,7 & 113 & 2,3 \\
Hemofílico & 11 & 0,3 & 0 & 0 & 11 & 0,2 \\
Transfusão & 3 & 0,1 & 3 & 0,2 & 6 & 0,1 \\
Transmissão Vertical & 26 & 0,8 & 34 & 2,2 & 60 & 1,2 \\
Ignorado & 1050 & 33,8 & 498 & 32,0 & 1548 & 33,7 \\
\hline
\end{tabular}

*UDI=Usuário de Drogas Injetáveis.

A Tabela 2 mostra que as principais categorias de exposição à AIDS no período analisado foram a heterossexual $(39,1 \%)$, seguida dos casos ignorados $(33,7 \%)$ e da homossexual $(15,1 \%)$.

A análise da incidência de AIDS, segundo categoria de exposição e sexo no período analisado, mostrou que no sexo masculino a principal categoria de exposição foi heterossexual $(26,8 \%)$, seguida de homossexual $(22,8 \%)$ e bissexual $(12,7 \%)$. Entre as mulheres também predominou a exposição heterossexual, com 995 casos $(63,9 \%)$.

\section{DISCUSSÃO}

O perfil epidemiológico dos casos de AIDS no Rio Grande do Norte, no período de 1980 a 2013, acompanhou a evolução da epidemia no Brasil, que apresentou três fases distintas: a primeira foi até 1986, caracterizada pelo predomínio da transmissão sexual entre os homossexuais do sexo masculino; a segunda fase da epidemia ocorreu entre o fim da década de 80 e início dos anos 90, na qual o uso de drogas injetáveis apareceu como principal forma de transmissão; e a terceira fase, entre o fim dos anos 90 até o momento atual, apresenta predomínio da prática heterossexual como forma de transmissão do vírus da AIDS para as mulheres. ${ }^{8}$

Destaca-se que nesta última fase, assim como em todo o período analisado, o número de casos diagnosticados de AIDS no Rio Grande do Norte apresentou-se crescente, com períodos de leve queda e diminuição importante dos casos entre 2012 e 2013. Esta situação pode ser atribuída ao fato dos dados disponíveis no DATASUS, referentes ao ano de 2013, encontrarem-se consolidados até o seu primeiro semestre.

Os dados do Boletim Epidemiológico Brasileiro mostram um ligeiro predomínio do sexo masculino e tendência à feminização, baixa escolaridade e renda, além de pessoas em idade economicamente ativa. Em 2012, a taxa de incidência de casos de AIDS em homens foi de $26,1 / 100.000$ habitantes e de 14,5 em mulheres, apresentando uma razão de sexos de 1,7 casos em homens para cada caso em mulheres, atingindo o seu menor valor no ano de $2005(1,4)$ e apresentando aumento desde então. ${ }^{2}$

A epidemia da AIDS no Rio Grande do Norte tem como característica predominante a transmissão sexual, indicando uma heterossexualização da epidemia, que pode ser evidenciada em vários estudos. ${ }^{9-11}$ Apesar do evidente avanço da doença entre os indivíduos heterossexuais, estudo internacional aponta elevada prevalência da infecção pelo HIV entre homens que fazem sexo com outros homens e transexuais $(10,6 \%$ e $17,7 \%$ respectivamente) em comparação à população geral (entre $0,4 \%$ a $1 \%)^{1}{ }^{1}$

Apesar de ainda haver mais casos de AIDS entre os homens do que entre as mulheres, essa diferença tem diminuído ao longo dos anos, indicando o avanço do processo 


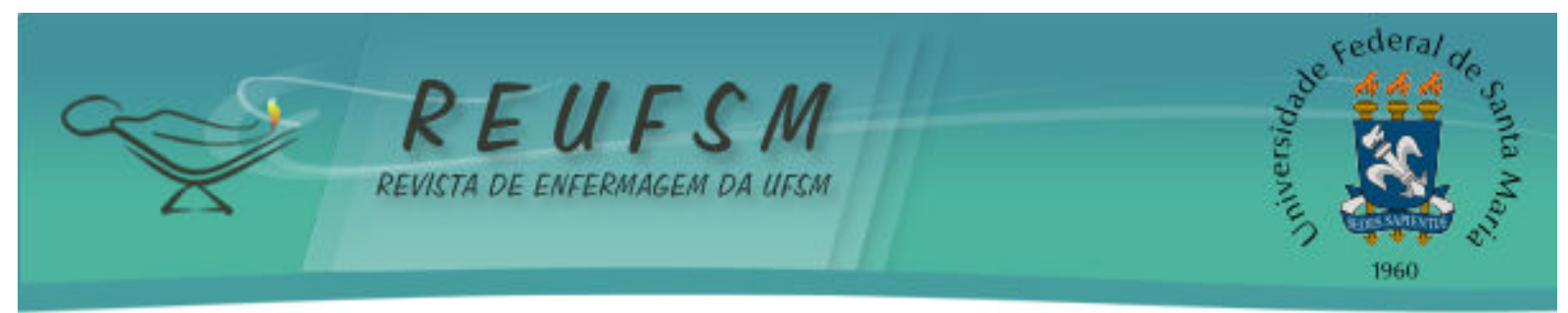

de feminização do HIV, no qual o número de mulheres infectadas aumenta rapidamente entre as heterossexuais casadas, com parceiro único e não-usuárias de drogas, sendo a relação sexual a principal via de transmissão do HIV para essas mulheres. ${ }^{12} \mathrm{Na}$ América Latina, 31\% dos adultos infectados pelo HIV são mulheres e nos países do Caribe esse número chega a $52 \%{ }^{1}$

Em estudo realizado em um município do Rio Grande do Norte ${ }^{13}$, encontrou-se que as mulheres pesquisadas citaram o preservativo como meio fundamental de se prevenir da infecção pelo HIV. No entanto, pelo fato de estarem casadas, essas mulheres não se consideram em situação de vulnerabilidade e não utilizavam o preservativo nas suas relações sexuais. As relações de gênero nas questões conjugais e sexuais ainda são evidentes. ${ }^{13}$

Com o maior número de mulheres com AIDS identificou-se o aumento de casos de crianças infectadas por meio da transmissão vertical do HIV. Assim, com o objetivo de prevenir a transmissão vertical do vírus no Brasil, recomenda-se oferecer o teste anti-HIV para todas as gestantes, mediante seu consentimento, com aconselhamento pré e pósteste, independente de apresentar situação de risco para a infecção pelo HIV. ${ }^{14}$

No Estado do Rio Grande do Norte, a faixa etária mais acometida pela AIDS está entre 20 e 49 anos. Esse dado assemelha-se a realidade do Brasil, que aponta que a AIDS é mais incidente, nos indivíduos, de ambos os sexos, entre 25 a 49 anos. Do total de casos identificados em homens, $78 \%$ estão nessa faixa etária, para as mulheres, essa proporção corresponde a $71 \%{ }^{15}$

Estudo que avaliou o comportamento sexual da população brasileira sexualmente ativa constatou que entre os indivíduos com vida sexual ativa, $28,5 \%$ deles estavam entre 20 e 29 anos, $27,2 \%$ entre 30 e 39 anos, e 20,2\% entre 40 e 49 anos. Verificou-se também que as pessoas entre 20 a 39 anos possuíam uma média de relações sexuais $47,8 \%$ maior do que a observada entre aqueles indivíduos de 14 a 19 anos, e o dobro da média daquelas acima de 50 anos de idade. ${ }^{16}$

No caso específico da região do Rio Grande do Norte, observou-se que a maior parte de sua população encontrava-se na faixa etária entre 20 a 49 anos, representando 46,74\%. Com relação às informações sobre o nível de escolaridade da população desse estado, identificou-se 47,48\% dos indivíduos apresentou-se sem instrução ou com nível fundamental incompleto. ${ }^{7}$ Esse aspecto regional está associado a um pior conhecimento sobre o HIV/AIDS, o que dificulta o entendimento dos riscos de transmissão, contribuindo para aumentar a incidência dos casos de AIDS, especialmente na população sexualmente ativa. ${ }^{17}$

A segunda faixa etária com maior percentual de casos diagnosticados de AIDS no Rio Grande do Norte abrange indivíduos com idade mais avançada, observou-se uma incidência menor entre os jovens. Nesse contexto, ressalta-se que os dados nacionais apresentam uma tendência de crescimento nas taxas de detecção da doença na faixa etária de 15 a 24 anos e entre indivíduos com 50 anos ou mais. ${ }^{2}$

Os adolescentes apresentam vários comportamentos que os expõem ao risco de adquirirem o vírus da AIDS, como relação sexual precoce, a não adesão ao uso de preservativos durante a atividade sexual, e o uso de bebidas alcoólicas ou outras drogas. ${ }^{18}$ De maneira semelhante, identifica-se a necessidade de desenvolvimento de ações de prevenção, diagnóstico e assistência direcionada especificamente para a terceira idade ${ }^{19}$, principalmente, pelo aumento da expectativa de vida da população brasileira, além do aumento da proporção de indivíduos mais velhos com infecção pelo HIV e, consequentemente, crescimento do número de casos de AIDS nesse público. ${ }^{20}$

Nesse sentido, as questões relacionadas à AIDS e ao envelhecimento devem ser aprofundadas, de modo a reorientar ações de prevenção e controle da infecção pelo HIV nessa faixa etária. 


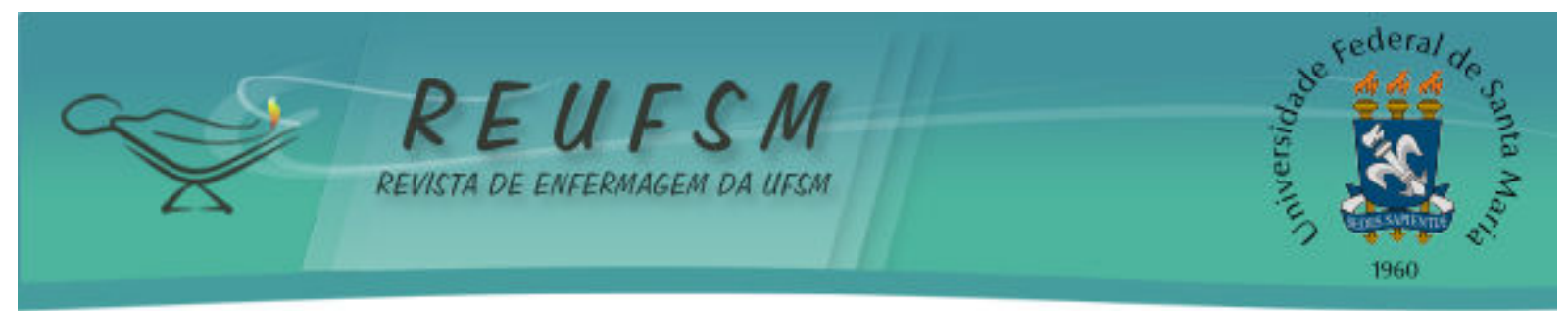

Outras questões têm evidenciado à tendência atual da epidemia de AIDS no cenário brasileiro, quais sejam: surgimento de epidemias microrregionais, com diferentes taxas de crescimento; diminuição das taxas de mortalidade, associada à introdução da terapêutica combinada antirretroviral no ano de 1996; progressiva expansão da doença para áreas mais distantes dos centros urbanos, de menor porte e mais pobres, e aumento dos casos entre os indivíduos com níveis de escolaridade mais baixos. ${ }^{21}$

0 maior número de casos de AIDS em indivíduos com ensino fundamental incompleto pode influenciar na compreensão das orientações referentes ao tratamento e cuidados necessários com a saúde. Em um levantamento realizado no Brasil entre os jovens, constatou-se que quanto menor o nível de escolaridade, maior o percentual de infectados pelo vírus da AIDS (prevalência de 0,17\% entre os indivíduos com ensino fundamental incompleto e $0,10 \%$ entre aqueles que possuem ensino fundamental completo) ${ }^{22}$

0 fenômeno da pauperização tem sido caracterizado pelo aumento da proporção de casos de AIDS em indivíduos de baixa escolaridade. 0 nível educacional expressa diferenças entre pessoas em termos de acesso à informação, perspectivas e possibilidades de se beneficiar de novos conhecimentos. É inegável que alguns parâmetros para alcançar maior qualidade de prevenção e assistência, tais como o acesso à educação e aos métodos preventivos, estão diretamente ligados à situação socioeconômica da população. ${ }^{23}$

Com relação à raça, excluídos os casos com campo ignorado, percebeu-se que há um alto índice de casos de AIDS notificados no Rio Grande do Norte presentes na raça parda, seguida da branca. Tal dado difere do cenário brasileiro, o qual mostra uma maior concentração dos casos de AIDS entre os brancos. ${ }^{2}$ Entretanto, nos últimos dez anos, observa-se uma tendência de crescimento da doença nos indivíduos pardos ${ }^{2}$. No Rio Grande do Norte, isso pode ser atribuído ao fato da maioria da população rio-grandense-do-norte ser composta por pessoas da raça parda. ${ }^{7}$

As melhorias no tratamento da AIDS, tanto medicamentoso como no acompanhamento dos infectados tem se mostrado mais eficaz, devido aos avanços nas políticas de saúde. No entanto, problemas como falha de adesão, resistência viral, retenção em rede de serviços especializados e o diagnóstico tardio tem contribuído para que os casos de AIDS continuem aumentando. ${ }^{2}$

Há a necessidade de melhorar o monitoramento da epidemia, equilibrar o financiamento das estratégias de prevenção e tratamento, direcionando-as para as populações de maior risco, ao possibilitar a continuidade e o monitoramento do cuidado. ${ }^{1}$ Faz-se fundamental adotar estratégias de educação em saúde e de apoio, por meio do cuidado de enfermagem e no acompanhamento interdisciplinar de saúde, a fim de ampliar a divulgação das informações e favorecer a autonomia do cuidado de si. ${ }^{20}$

Como limitações deste estudo, pelo uso de dados secundários, identificaram-se várias informações como categoria de exposição e raça ignoradas nos casos notificados de AIDS no Estado do Rio Grande do Norte. Um dos principais problemas da vigilância epidemiológica é a baixa qualidade da informação coletada e a subnotificação de casos de AIDS. Nesse sentido, faz-se importante a completude e a atualização dos dados de notificação, melhorando a qualidade e confiabilidade desses dados de forma a contribuir com o planejamento das ações em HIV/AIDS. ${ }^{19}$

\section{CONCLUSÃO}

0 perfil dos casos de AIDS diagnosticados no Rio Grande do Norte, no período de 1980 a 2013, é semelhante ao perfil epidemiológico brasileiro, com predomínio de pessoas do sexo masculino, em idade economicamente ativa e com baixa escolaridade. 


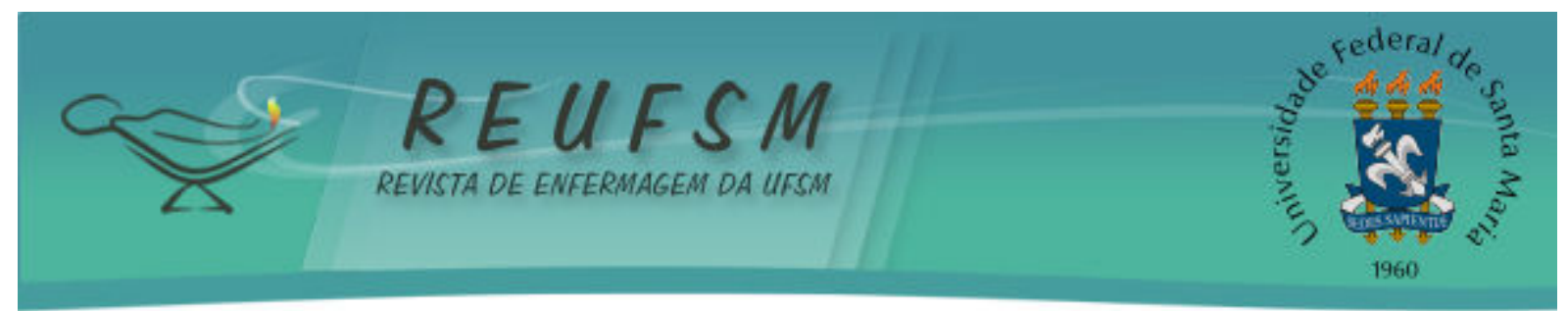

No período analisado, a faixa etária mais acometida pela AIDS foi entre 20 e 49 anos, indivíduos com ensino fundamental incompleto, raça parda e predomínio do sexo masculino com aumento crescente dos casos em pessoas do sexo feminino. A categoria de exposição mais frequente foi a de heterossexuais tanto para o sexo masculino quanto para o sexo feminino. Identifica-se que os casos da doença no Rio Grande do Norte apresentam a feminização, a heterossexualização e a baixa escolaridade como características marcantes.

Evidenciou-se que os dados provenientes do DATASUS, além de apresentarem fácil acessibilidade, forneceram informações relevantes que possibilitaram traçar um perfil epidemiológico dos casos de AIDS diagnosticados no Rio Grande do Norte. Apesar disso, a existência de dados ignorados representou um déficit de informações, refletindo em uma limitação do sistema e, consequentemente, uma limitação do estudo.

Pesquisas como esta podem contribuir para o direcionamento de ações de prevenção, promoção e controle do HIV/AIDS e também para adotar políticas adequadas com relação à transmissão, diagnóstico e tratamento. Assim, conhecer as características epidemiológicas loco-regionais pode proporcionar o planejamento de ações de saúde específicas.

Salienta-se que a enfermagem, como integrante da equipe multiprofissional, acaba, muitas vezes, assumindo a tarefa de coordenar a equipe de saúde. Nesse contexto, a análise da situação epidemiológica torna-se um instrumento importante no processo de gerenciamento dos serviços e na melhoria da qualidade da assistência de enfermagem.

Assim, o enfermeiro, como outros profissionais de saúde, não devem se limitar apenas às questões clínicas, mas também levar em consideração aspectos gerais, como a situação do local onde desenvolvem o seu processo de trabalho, reforçando a importância de compreender as implicações das diferentes variáveis que estão relacionadas ao processo de saúde e adoecimento, como um recurso para o planejamento do cuidado.

Nesse sentido, faz-se necessário continuar a aprofundar os estudos sobre a temática da AIDS e o uso de dados dos sistemas de informação em saúde, de modo a proporcionar também uma avaliação da organização e disponibilidade desses dados, com vistas a atender às necessidades da pesquisa e da assistência à saúde.

\section{REFERÊNCIAS}

1. García PJ, Bayer A, Cárcamo CP. The changing face of HIV in Latin America and the Caribbean. Curr HIV/AIDS Rep. 2014;11(2):146-57.

2. Boletim Epidemiológico Aids/DST [Internet]. 2013 [acesso em 2014 jun 25];(1):1-60. Disponível em: http://www.aids.gov.br/publicacao/2013/boletim-epidemiologico-aids-edst-2013.

3. Pereira BS, Silva JK, Marques MS, Soares HC. Enfrentamento da feminização da epidemia da AIDS. FG Ciência. 2011;1(1):1-18.

4. Porto TSAR, Silva CM, Vargens OMC. Cuidando de mulheres com HIV/AIDS: uma análise interacionista na perspectiva de mulheres profissionais de saúde. Rev Gaúch Enferm [Internet]. 2014 [acesso em 2014 jul 9];35(2):40-6. Disponível em: http://seer.ufrgs.br/index.php/RevistaGauchadeEnfermagem/article/view/41253.

5. Grangeiro A, Silva LL, Teixeira PR. Resposta à AIDS no Brasil: contribuições dos movimentos sociais e da reforma sanitária. Rev Panam Salud Pública. 2009;26(1):87-94.

6. Grangeiro A, Escuder MML, Castilho EA. A epidemia de AIDS no Brasil e as desigualdades regionais e de oferta de serviço. Cad Saúde Pública [Internet]. 2010 [acesso em 2014 jun 


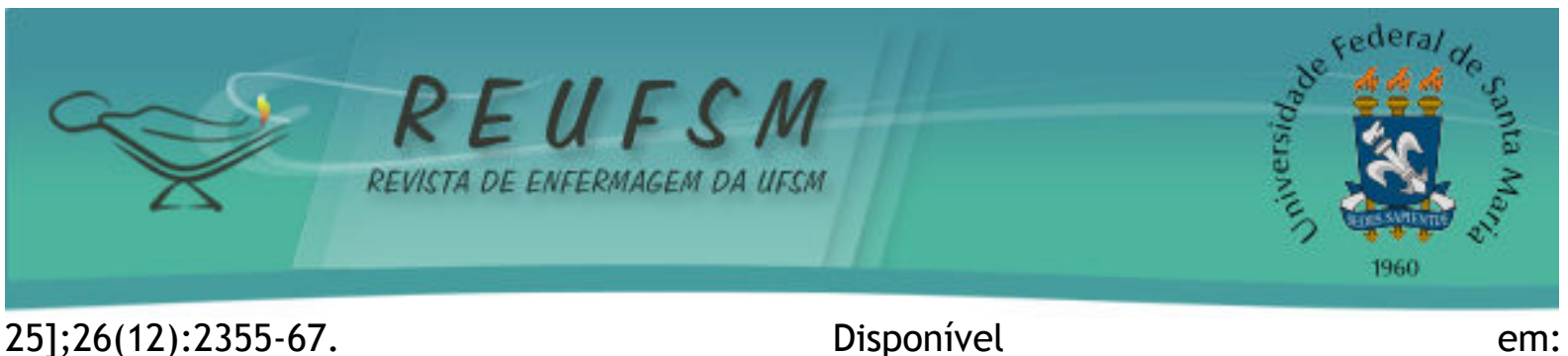

http: / /www.scielo.br/scielo.php?script=sci_arttext\&pid=S0102-

311X2010001200014\&lng=pt.

7. Instituto Brasileiro de Geografia e Estatística (IBGE). Estados: Rio Grande do Norte [Internet]. Brasília (DF): IBGE; 2013 [acesso em 2014 jun 14]. Disponível em: http: / /www.ibge.gov.br/estadosat/perfil.php?sigla=rn.

8. Santos NJS, Barbosa RM, Pinho AA, Villela WV, Aidar T, Filipe EMV. Contextos de vulnerabilidade para o HIV entre mulheres brasileiras. Cad Saúde Pública [Internet]. 2009 [acesso em 2014 jun 12]; 25 Supl 2:321-33. Disponível em: http://www.scielo.br/pdf/csp/v25s2/14.pdf.

9. Reis RK, Santos CB, Dantas RAS, Gir E. Qualidade de vida, aspectos sociodemográficos e de sexualidade de pessoas vivendo com HIV/AIDS. Texto \& Contexto Enferm [Internet]. 2011 [acesso em 2014 maio 16];20(3):565-75. Disponível em: http: / / www.scielo.br/pdf/tce/v20n3/19.pdf.

10. Fiuza MLT, Lopes EM, Alexandre HO, Dantas PB, Galvão MTG, Pinheiro AKB. Adesão ao tratamento antirretroviral: assistência integral baseada no modelo de atenção as condições crônicas. Esc Anna Nery Rev Enferm [Internet]. 2013 [acesso em 2014 jun 25];17(4):740-8. Disponível em: http://www.scielo.br/scielo.php?script=sci_arttext\&pid=S1414$81452013000400740 \& \operatorname{lng}=$ pt.

11. Simon D, Béria JU, Tietzmann DC, Carli R, Stein AT, Lunge VR. Prevalência de subtipos do HIV-1 em amostra de pacientes de um centro urbano no sul do Brasil. Rev Salud Pública [Internet]. 2010 [acesso em 2014 maio 18];44(6):1094-101. Disponível em: http: / / www.scielo.br/scielo.php?script=sci_arttext\&pid=S0034-89102010000600015.

12. Carneiro AJS, Coelho, EAC. Aconselhamento na testagem anti-HIV no ciclo gravídicopuerperal: o olhar da integralidade. Ciênc Saúde Coletiva [Internet]. 2010 [acesso em 2014 jun 20];15 Supl 1:1216-26. Disponível em: http://www.scielo.br/scielo.php?pid=S141381232010000700031 \&script=sci_arttext.

13. Figueredo LG, Silva RAR, Silva ITS, Souza KGS, Silva FFA. Percepção de mulheres casadas sobre o risco de infecção pelo HIV e o comportamento preventivo. Rev Enferm UERJ [Internet]. 2013 [acesso em 2014 maio 22];21(Esp 2):805-11. Disponível em: http: / /www.facenf.uerj.br/v21esp2/v21e2a18.pdf.

14. Araújo CLF, Pacheco CC, Lins S, Bastos VD, Loureiro TPC, Silva LOG. Oferta do teste rápido para HIV em maternidades: a visão das mulheres. Rev Enf Profissional [Internet]. 2014 [acesso em 2014 jun 19];1(1):20-36. Disponível em: http://www.seer.unirio.br/index.php/enfermagemprofissional/article/viewFile/3510/pdf_ 1199.

15. Brasil. Ministério da Saúde. Secretaria de Vigilância em Saúde. Departamento de DST/AIDS e Hepatites Virais. Recomendações para profilaxia da transmissão vertical do HIV e terapia antirretroviral em gestantes. Brasília (DF): Ministério da Saúde; 2010. 146 p.

16. Programa Nacional de DST/Aids. Comportamento da população brasileira sexualmente ativa [Internet]. 2003 [acesso em 2014 nov 2]. Disponível em: http: / / www.aids.gov.br/sites/default/files/briefing2.pdf.

17. Irffi G, Soares RB, Souza SA. Fatores Socioeconômicos, Demográficos, Regionais e Comportamentais que Influenciam no Conhecimento sobre HIV/AIDS. EconomiA [Internet]. 


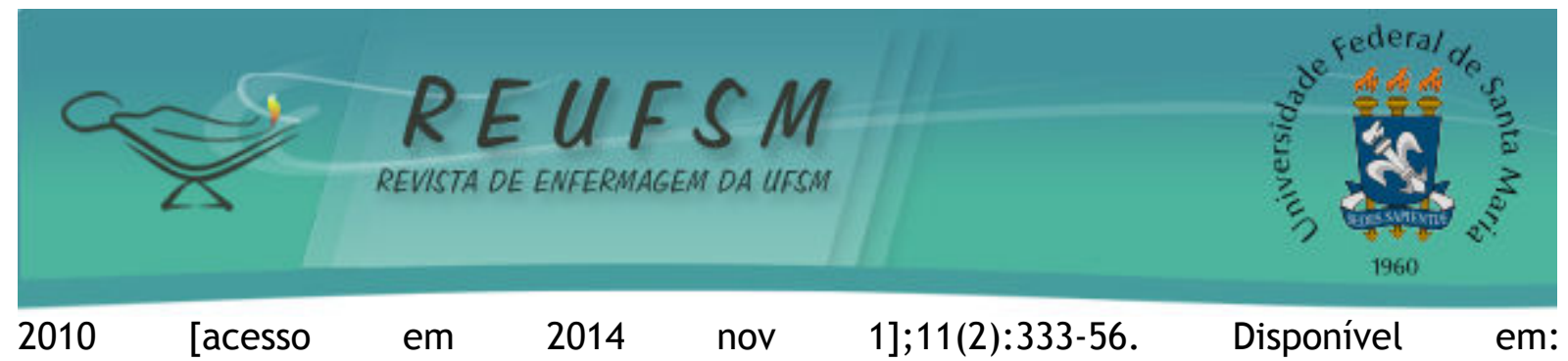

http://www.anpec.org.br/revista/vol11/vol11n2p333_356.pdf.

18. Araújo TME, Monteiro CFS, Mesquita GV, Alves ELM, Carvalho KM, Monteiro RM. Fatores de risco para infecção por HIV em adolescentes. Rev Enferm UERJ [Internet]. 2012 [acesso em 2014 jun 13];20(2):242-7. Disponível em: http://www.facenf.uerj.br/v20n2/v20n2a17.pdf.

19. Silva MM, Vasconcelos ALR, Ribeiro LKNP. Caracterização epidemiológica dos casos de AIDS em pessoas com 60 anos ou mais, Pernambuco, Brasil, 1998 a 2008. Cad Saúde Pública [Internet]. 2013 [acesso em 2014 jun 25];29(10):2131-5. Disponível em: http: / /www.scielo.br/scielo.php?script=sci_arttext\&pid=S0102-

311X2013001000028\&lng=pt.

20. Melo HMA, Leal MCC, Marques APO, Marino JG. O conhecimento sobre Aids de homens idosos e adultos jovens: um estudo sobre a percepção desta doença. Ciênc Saúde Coletiva [Internet]. 2012 [acesso em 2014 nov 1];17(1):43-53. Disponível em: http://www.scielo.br/pdf/csc/v17n1/a07v17n1.pdf.

21. Souza CC, Mata LRF, Azevedo C, Gomes CRG, Cruz GECP, Toffano SEM. Interiorização do HIV/AIDS no Brasil: um estudo epidemiológico. Rev Bras Ciênc Saúde [Internet]. 2013 [acesso em 2014 jun 12];11(35):25-30. Disponível em: http://seer.uscs.edu.br/index.php/revista_ciencias_saude/article/view/1798/1380.

22. Departamento de DST, Aids e Hepatites Virais. Aids no Brasil [Internet]. [acesso em 2014 jun 12]. (cerca de 3 telas). Disponível em: http://www.aids.gov.br/pagina/aids-nobrasil.

23. Pottes FA, Brito AM, Gouveia GC, Araújo EC, Carneiro RM. Aids e envelhecimento: características dos casos com idade igual ou maior que 50 anos em Pernambuco, de 1990 a 2000. Rev Bras Epidemiol [Internet]. 2007 [acesso em 2014 jun 21];10(3):338-51. Disponível em: http://www.scielo.br/scielo.php?pid=\$1415-790X2007000300005\&script=sci_arttext.

24. Paula CC, Padoin SMM, Albuquerque PVC, Bubadué RM, Silva CB, Brum CN. Cotidiano de adolescentes com o vírus da imunodeficiência humana em tratamento. Rev Enferm UFSM [Internet]. 2013 [acesso em 2014 jun 21];3(3):500-8.Disponível em: http://cascavel.ufsm.br/revistas/ojs-2.2.2/index.php/reufsm/article/view/9862.

Data de recebimento: $18 / 08 / 2014$

Data de aceite: $26 / 11 / 2014$

Contato com autor responsável: Ilisdayne Thallita Soares da Silva

Endereço postal: Rua Nossa Senhora do Desterro, 237, Bairro Conjunto Cônego Monte, CEP 59200-000, Santa Cruz, Rio Grande do Norte, Brasil.

E-mail: ilisdayne@yahoo.com.br 